\title{
Erythema Multiforme Minor with Rare Etiology
}

\author{
Rashmi. J. Kurup*, Vathsala Naik MDS, Amandeep Sodhi MDS \\ Department of Oral Medicine and Radiology, Bangalore Institute of Dental Sciences, Bangalore, India
}

Received June 22, 2020; Revised July 24, 2020; Accepted August 25, 2020

\section{Cite This Paper in the following Citation Styles}

(a): [1] Rashmi. J. Kurup, Vathsala Naik MDS, Amandeep Sodhi MDS , "Erythema Multiforme Minor with Rare Etiology," Open Journal of Dentistry and Oral Medicine, Vol. 8, No. 2, pp. 17 - 21, 2020. DOI: 10.13189/ojdom.2020.080202.

(b): Rashmi. J. Kurup, Vathsala Naik MDS, Amandeep Sodhi MDS (2020). Erythema Multiforme Minor with Rare Etiology. Open Journal of Dentistry and Oral Medicine, 8(2), 17 - 21. DOI: 10.13189/ojdom.2020.080202.

Copyright@2020 by authors, all rights reserved. Authors agree that this article remains permanently open access under the terms of the Creative Commons Attribution License 4.0 International License

\begin{abstract}
Erythema Multiforme is an acute, selflimiting inflammatory mucocutaneous condition caused by a hypersensitivity reaction with distinctive clinical eruption manifested as iris or target lesions. It is a life-threatening mucocutaneous disorder where early diagnosis and management are of utmost importance. Erythema Multiforme results from a cell-mediated immune reaction against a precipitating factor. It is clinically characterized by a "minor" form and a "major" form. In the Erythema Multiforme minor, only one mucous membrane is affected and usually is the oral mucosa. Erythema Multiforme is diagnosed based on stringent clinical findings which are pathognomic as microscopic evaluation carries the least significance. The range of possible etiologies for the oral disease is immense including infectious agents, drugs, food additives, etc. We report a case of a 68-year-old female who presented with a complaint of painful ulcers and burning sensation in her mouth making her difficult to eat \& drink. History revealed the usage of clove to get rid of tooth pain following which she developed ulcers in the mouth. In the present case, based on patient history, the clove was found to be a probable etiological agent. The patient was successfully treated with corticosteroids adhering to systemic corticosteroid administrative protocols and no remissions and exacerbations were noticed in 6 months follow up. Early diagnosis of the disease remains essential to promptly initiate appropriate management and proper follow up. Recognition and withdrawal or prevention of contact with the etiological agent is an important step in the management of EM. In the present case, the clove was found to be a probable etiological agent.
\end{abstract}

Keywords Erythema Multiforme, Target Lesions, Iris
Lesions, Steven Johnson Syndrome, Toxic Epidermal Necrolysis, Corticosteroids

Keywords EM- Erythema Multiforme, EMMErythema multiforme major, EMm- Erythema multiforme minor, SSJ- Steven Johnson syndrome, TEN- Toxic Epidermal necrolysis.

\section{Introduction}

Erythema multiforme (EM) is an intense, self-limiting, sometimes recurrent mucocutaneous disorder that manifests with a reaction pattern that appears as a consequence of allergic host response to an antigenic challenge. It usually affects individuals at 20 to 40 years [1]. In the minor form oral cavity is frequently affected. The prevalence of oral EM minor varies from 35\% to $65 \%$ among patients with skin lesions.

An inter-disciplinary study reported that $70 \%$ of cutaneous EM minor patients had an oral involvement, comprising of multiple, large, shallow, extremely painful, and debilitating ulcers [2]. The oral lesions have a predilection for the vermilion border of the lips and the buccal mucosa, mostly sparing the gingiva.

The exact pathogenesis of EM is still unknown and it has been postulated that EM results from T-cell-mediated immune reaction to the precipitating etiological agent leading to a cytotoxic immunological attack on keratinocytes that express non-self-antigens causing vesicles, blisters and erosions [3]. It is a disease of abrupt onset involving skin and mucous membranes where the lesions appear as red macules, papules, and vesicles. 
Blistering and crusting may occur in the center of the lesion resulting in the characteristic iris or target lesions.

We present a case of condiment induced EM minor and believe that the inducing agent is clove which is otherwise used for relief from tooth pain.

\section{Case Report}

A 68-year-old female reported to the Department of Oral Medicine and Radiology, with a complaint of painful ulcers and burning sensation in her mouth for 3 days. (Figure 1). History of tooth pain (lower front tooth region) from 2 weeks. No relief from medications due to which she started using clove for her pain. She is known hypertensive from past 5 years and is under medication for the same

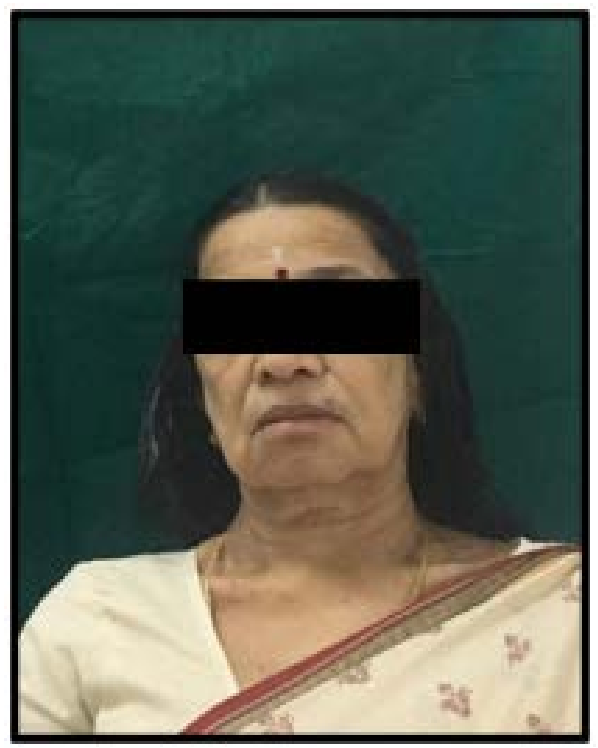

Figure 1. A 68- year -old female patient on the day of reporting to the Department of Oral Medicine and Radiology

After the third day of using the clove, she noticed ulcers in her mouth making her difficult to consume solid and liquid foods due to pain. No evidence of target lesions was noticed on the extremities.

Hemorrhagic crustations with clinically evident bleeding were seen on the lower lip. (Figure 2)

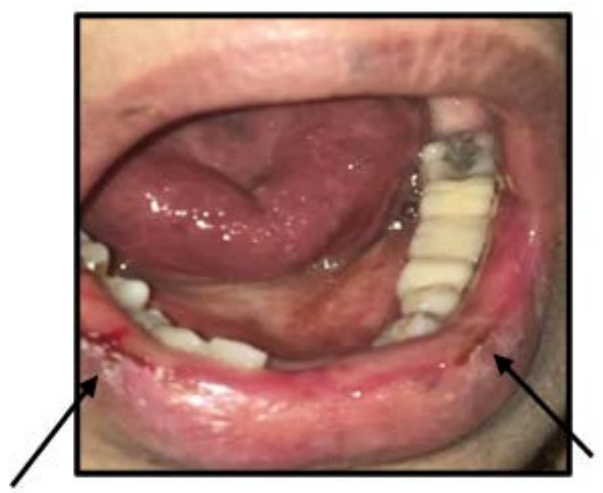

Figure 2. Hemorrhagic crustations with clinically evident bleeding
Intraorally diffuse redness and multiple small macules (lower labial mucosa) were noticed. (Figure 3)

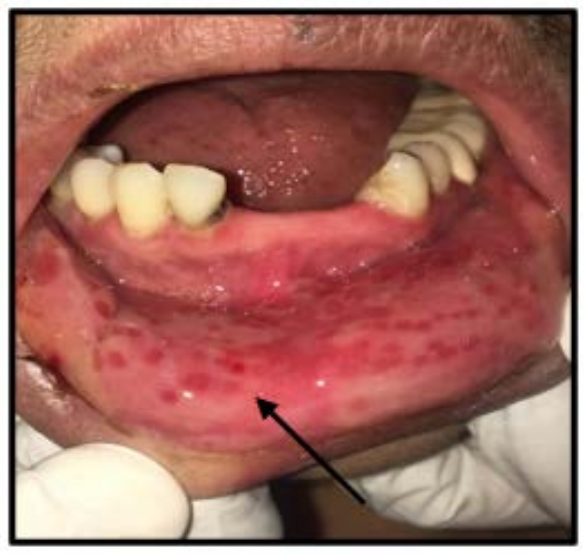

Figure 3. Diffuse redness and multiple small macules

Discrete areas of sloughing in the right buccal mucosa (Figure 4) and left buccal mucosa (Figure 5) which was tender with no associated bleeding.

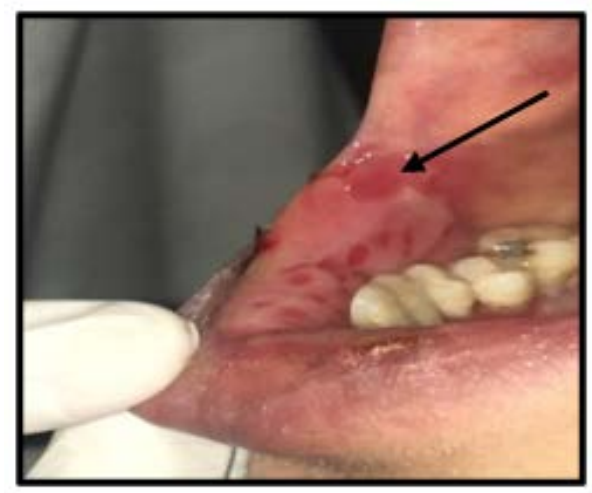

Figure 4. Discrete areas of sloughing in the right buccal mucosa

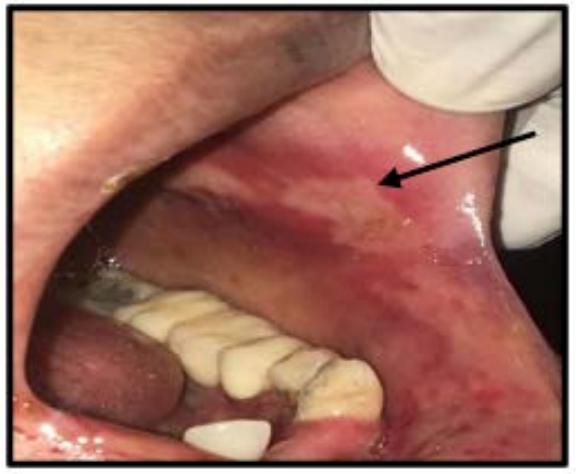

Figure 5. Discrete areas of sloughing in the left buccal mucosa

Based on the typical clinical presentation of the disease, a provisional diagnosis of erythema multiforme -minor was given.

Clinical differential diagnosis included Allergic reactions, Herpetic gingivostomatitis, Mucous membrane pemphigoid, Pemphigus Vulgaris, Erosive lichen planus, and Recurrent aphthous ulcers. 
Along with symptomatic treatment for her toothache, the patient was advised anti-histaminic mouth wash. Systemic corticosteroids were started with a dose of prednisolone 20 mg t.i.d for a week followed by tapering dosages and systemic antifungal fluconazole $150 \mathrm{mg}$ once a week for 2 weeks were given. The patient was subjected to quadrant scaling, and oral hygiene instructions were given. After 3 weeks, the absence of hemorrhagic crustations with no bleeding was seen on the lower lip (Figure 6).

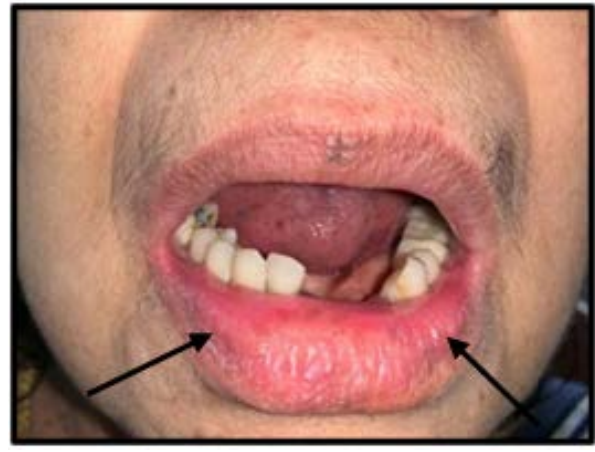

Figure 6. Absence of hemorrhagic crustations and bleeding

Intraorally with the absence of multiple small macules and redness were noticed on lower labial mucosa. (Figure 7)

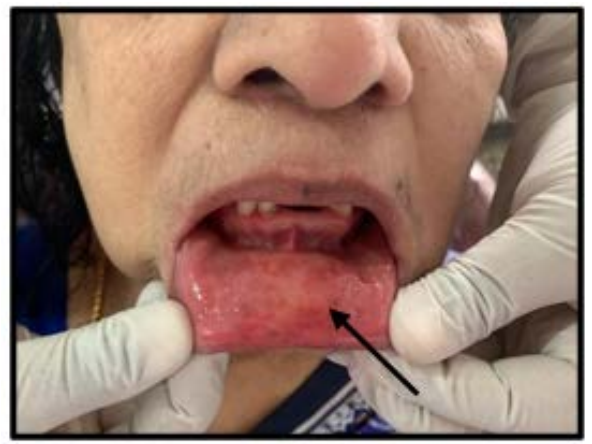

Figure 7. Absence of multiple small macules and redness

There were no discrete areas of sloughing in the right buccal mucosa (Figure 8) and left buccal mucosa (Figure 9) with no tenderness or associated bleeding.

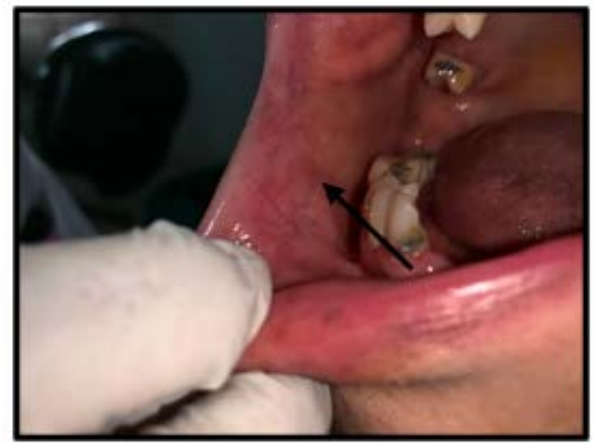

Figure 8. No discrete areas of sloughing in the right buccal mucosa

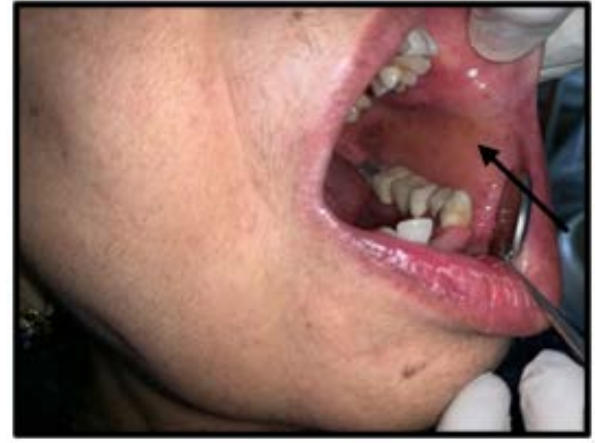

Figure 9. No discrete areas of sloughing in the left buccal mucosa

The patient is currently under review with no recurrence at 6 months follow-up. (Figure 10)

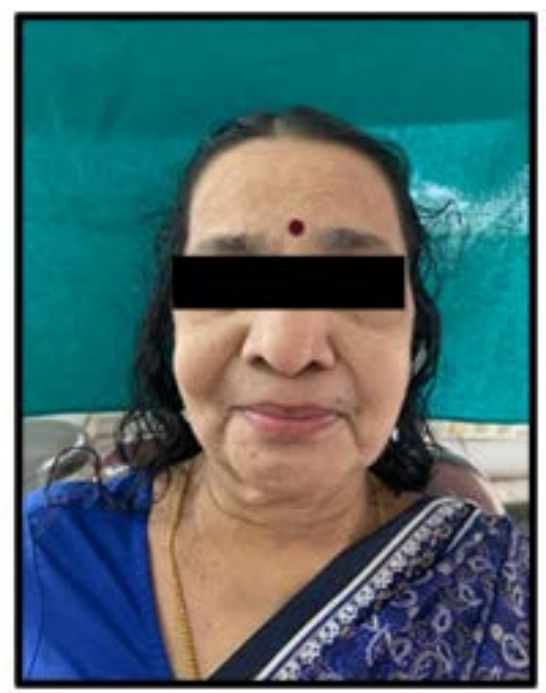

Figure 10. A 68-year-old female patient at 6 months follow-up

\section{Discussion}

Erythema multiforme was first described in 1866 by Hebra as a skin disease with symmetrically distributed red papules, evolving within several days to form annular or iris-like shapes or blisters. The term multiforme described this evolution of primary lesions into different forms [4].

It is a typically mild, self- limiting and sometimes recurring mucocutaneous reaction characterized by target or iris lesions of the skin or mucous membranes which resolve within 1 to 6 weeks. The best -documented trigger factor is drugs and microorganisms in $80-90 \%$ of cases [5].

It has been classified into many variants, mainly minor and major forms. EM minor (EMm) is mainly a cutaneous disease. Typical and/or raised atypical target lesions are the hallmark. Mucosal involvement is uncommon, when present, only one site is affected, most commonly the mouth. EMm that only affects the oral mucosa may occasionally arise. EM major (EMM) typically involves two or more mucous membranes with more variable skin involvement. Less than $10 \%$ of the body surface area but more severe than EMm is involved. Oral lesions are usually 
widespread and severe [6].

Oral involvement is estimated to occur with $25 \%-60 \%$ of patients with erythema multiforme. The severity of disease and the location of the lesions help to further differentiate into its major and minor forms. In contrast, erythema multiforme minor often presents with minimal to no mucosal membrane involvement and milder cutaneous symptoms.

Diseases that have similar clinical features as that of erythema multiforme include Stevens-Johnson syndrome (SSJ), and Toxic Epidermal Necrolysis (TEN) that have a higher mortality rate. The disease is classified as Stevens-Johnson when the generalized vesicles and bullae involve the skin, mouth, eyes and genitals. The most severe form of the disease is TEN, which is usually secondary to a drug reaction and results in sloughing of skin and mucosa in large sheets. Morbidity is high from secondary infection [6].

\section{Box 1 - Most Common Associations with Erythema Multiforme [7]}

\section{Etiological agents}

\section{Micro-organisms}

Viruses: herpes viruses (HSV, VZV, EBV), hepatitis viruses (A, B and C)

Bacteria: Mycoplasma pneumoniae, Chlamydia, Corynebacterium diphtheria

Fungi and parasites: coccidioidomycosis, histoplasmosis

2. Drugs: allopurinol, barbiturates, cancer chemotherapeutic agents, carbamazepine, cephalosporins, non-steroidal anti-inflammatory drugs, penicillin's, phenytoin.

3. Food additives or chemicals: benzoates, nitrobenzene, terpenes, ethanol

4. Immune and other conditions: graft versus host disease, immunization (BCG, hepatitis B), sarcoidosis, systemic lupus erythematous

Flow Chart - I

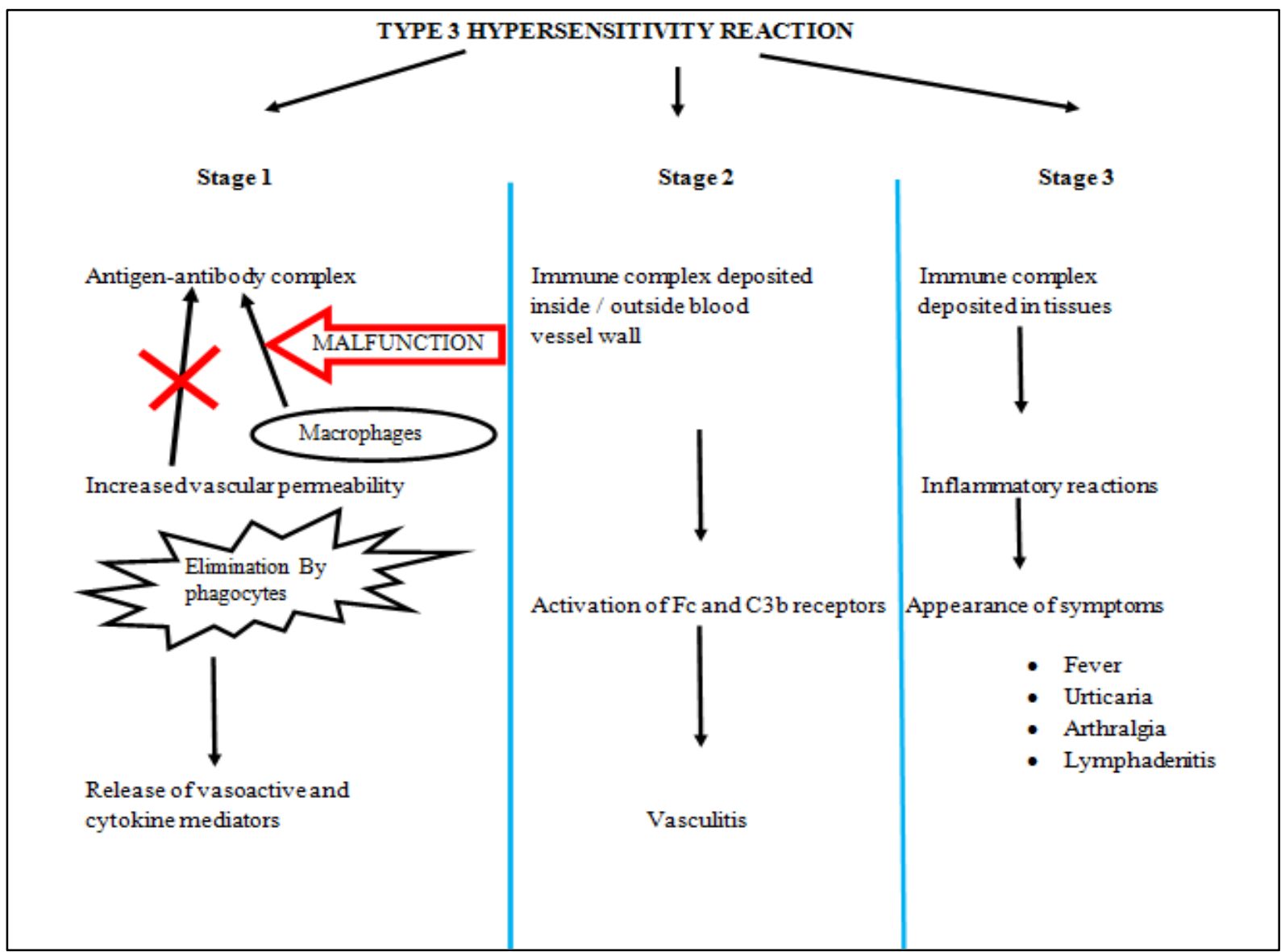


There are no specific diagnostic tests for EM and the diagnosis is mainly clinically supported if necessary, by biopsy [8].

Oral lesions have different clinical presentations, ranging from diffuse oral erythema, to multifocal superficial ulcerations, thus the term multiforme. Initially, vesicles or bullae may be present, which rupture causing hemorrhagic crustations. Any area of the mouth may be involved, with buccal mucosa, palate, and tongue being most frequently affected. In most cases, lip lesions show hemorrhagic crustations. There may be mild to severe oral and perioral pain that may interfere with functional activities like speech, eating, swallowing, and fluid intake, debilitating the health of the patient. Intraoral and perioral lesions heal without scarring, usually mild cases heal within 2-4weeks with local debridement, plenty of fluid intake, and topical analgesics. Oral antihistamines and topical steroids provide symptomatic relief [2].

Ramlal et al reported a case of a 38-year-old male who presented with a complaint of severe oral \& cutaneous lesions making him difficult to eat \& drink. History revealed the usage of clove to get rid of tooth pain following which he developed ulcers in the mouth which made him visit a general physician where he was administered gentamycin. Later on, oral lesions worsened along with the emergence of dermal lesions. Based on the patient history, clove was found to be a probable etiological agent and the condition was further precipitated owing to the administration of gentamycin. The patient was successfully treated with corticosteroids adhering to systemic corticosteroid administrative protocols and no remissions and exacerbations were noticed in a year follow up [3].

Before any treatment is prescribed, possible underlying causes, such as medications, diet, infections, or systemic diseases should be determined and eliminated [7].

Management of EM also depends on many factors like disease severity, presence of mucosal disease, and recurrence. Administration of antiviral agents is of therapeutic value in herpes associated EM. Symptomatic treatment is achieved with topical analgesics and mouth rinses in addition to the intake of a soft bland diet. Corticosteroids are the most commonly used drugs in the management of EM. In the present case, the lesions regressed after corticosteroid therapy.

\section{Conclusions}

Erythema multiforme being a reactive ulcerative lesion is multifactorial in origin. Recognition and withdrawal or prevention of contact with the etiological agent is an important step in the management of EM. In the present case, the clove was found to be a probable etiological agent. There is no precise diagnostic investigation for EM. The vital clues to diagnosis continue to be the clinical history and clinical findings. Corticosteroids are the mainstay for the management of the disease even after lack of any clinical evidence. Early diagnosis of the disease remains essential to promptly initiate appropriate management and proper follow up. Oral and maxillofacial physicians play a pivotal role in early diagnosis and prompt management of this debilitating condition.

\section{REFERENCES}

[1] Rashmitha A, Gotoor SG, Muppirala S, Raju DR. Erythema multiforme: A case report. Journal of Indian Academy of Oral Medicine and Radiology. 2017 Apr 1;29(2):153.

[2] Shrihari TG, Shetty SR. Erythema multiforme: A mysterious lesion!. Indian Journal of Medical and Paediatric Oncology. 2018 Jul 1;39(3):363.

[3] Gantala R, Devaraju RR, Goud S, Aitha H, Kubbi JR. Condiments costing high...! A case report of erythema multiforme. Journal of clinical and diagnostic research: JCDR. 2015 Jul;9(7):ZD22.

[4] Kroonen LM. Erythema multiforme: case report and discussion. The Journal of the American Board of Family Practice. 1998 Jan 1;11(1):63-5.

[5] Gupta N, Bansal A, Bansal A. Erythema Multiforme-Case Report with Review. Dental Journal of Advance Studies. 2014 Dec;2(03):166-8.

[6] Paulino L, Hamblin DJ, Osondu N, Amini R. Variants of Erythema Multiforme: A Case Report and Literature Review. Cureus. 2018 Oct;10(10).

[7] Kohli PS, Kaur J. Erythema multiforme-oral variant: case report and review of literature. Indian Journal of Otolaryngology and Head \& Neck Surgery. 2011 Jul 1;63(1):9-12.

[8] Bashir T, Arti S, Jyoti G, Monu Y, Vijay K, Naeem A. Erythema Multiforme: A Case Report. Int J Sci Stud. 2014;2(7):249-51. 